\title{
Die Arten der Gattung Balioptera
}

\author{
vom
}

Director Dr. Loew in Meseritz.

$\mathbf{M}_{\text {e }}$

eigen hat in der Gattung Opomyza Arten zusammengestellt, welche so wesentliche plastische Unterschiede zeigen, dafs ihre Vereinigung in eine einzige Gattung völlig unmöglich ist. Behält man für die Arlen aus dem nächsten Verwandtschaftskreise der Op. florum und Op. germinationis nach Zetterstedt's Vorgange den Gattungsnamen Opomyza bei, so bleiben immer noch Arten übrig, welche sich nicht in eine Gattung zusammenfassen lassen. Einige derselben gehören in die Gattung Sciomyza, andere in die Gattung Geomyza, auf eine derselben ist die Gattung Mycetaulus begründet u. s. w. Der nach allen diesen bereits vorgenommenen Absonderungen ver bleibende Rest wartet noch der systematischen Ordnung. Es befinden sich unter diesem Reste die 4 von Meigen unmittelbar hinter einander beschriebenen und einander in jeder Beziehung äufserst nahe verwandten Arten: Op. combinata, apicalis, venusta und tripunctata, welche den Stamm einer eigenen Gattung bilden, welche ich Balioptera nennen will.

Die wesentlichsten Merkmale der Gattung Balioptera sind folgende. Körperform schmal und gestreckt. Die Stirn trägt aufser den auf dem Scheitel befindlichen Borsten am oberen Theile ihres Seitenrands eine einzige aufwärtsgewendele Borste von grofser Länge. Fübler kurz, dem Gesicht aufliegend; die beiden ersten Glieder sehr kurz, das zweite auf der Oberseite mit einem aufgerichteten borstenartigen Haare, aber ohne vorwärtsgerichtete Borste; das dritte Glied rundlich oval, aufser seiner ganz kurzen mikroskopischen Pubescenz sparsam mit etwas längeren, schon bei mäfsiger Vergröfserung leicht bemerkbaren Härchen besetzt. Fühlerborste kurz behaart, auf ihrer Oberseite von längeren Haaren gekämmt.

Berl. Entomol. Zeitschr. VIII. 
Augen grofs, etwas unterhalb der Fühler einander am nächsten tretend. Backen schmal, mit Borsten beselzt, deren vorderste eine starke Knebelborste darstellt. Mundöffnung, Taster und Rüssel von mäfsiger Gröfse. Thoraxrücken bis vorn hin beborstet. Brustseiteu ohne Borste über den Vorderhüften, aber mit einer ansehnlichen Borste über den Miltelhüften. Schildchen an der Spitze mit zwei langen Borsten, vor denen jederseils in der Nähe des Seitenrands eine kürzere Borste steht. Beine ziemlich schlank; Mittelschienen deutlich gespornt; alle Schienen ohne aufgerichtetes Borstchen vor dem Ende ihrer Oberseite. Flügel schmal, gegen die Basis hin zugespitzt, ohne vortretenden Hinterwinkel und ohne deutliche Alula Die erste Längsader sehr kurz; nicht weit vor ihrem Ende hat sie eine Brechung, bis gegen welche hin sie von der Hülfsader begleitet wird, welche sich indessen nicht vollständig mit ihr verbindet; das letzte, kurze Ende der ersten Längsader bildet eine deutliche, mit ihrer Concavität nach vorn gerichtete Curve. Zweite Längsader auffallend lang, an ihrem Ende mit der in die Flügelspitze mündenden dritten Längsader etwas convergirend. Die kleine Querader steht weit jenseit der Mündung der ersten Längsader und die hintere Querader ist dem Flügelrande genähert. Die beiden kleinen Wurzelzellen sind vorhanden, doch ist das Vorderende der Querader, welche die vordere derselben von der Discoidalzelle trennt, völlig obliterirt. Unter der Behaarung des zweiten Abschnitts des Vorderrands finden sich keine stärkeren Borstchen.

Dafs ein Zusammenwerfen der Balioptera- und Geomyza-Arten völlig unstatthaft ist, ergiebt nachfolgende Zusammenstellung der wesentlichsten Unterschiede beider Gattungen zu vollster Genüge.

\section{Balioptera.}

Körperbau sehr schlank.

Am Seitenrande der Stirn eine Borste.

Fühler niederliegend.

Fühlerbors te auf der Oberseite gekämmt.

Schienen ohne aufgerichtetes Borstchen vor dem Ende der Oberseite.

Flügel gegen die Basis hin keil förmig zugespitzt, ohne vor-
Geomyza.

Körperbau nicht sehr schlank. Am Seitenrande der Stirn zwei Borsten.

Fühler abstehend.

Fühlerborste nur mit sehr kurzer Pubescenz.

Schienen mit anfgerichtetem Borstchen vor dem Ende der Oberseite.

Flügel gegen die Basis hin nicht keilförmig zugespitzt, mit stark 
tretenden Hinterwinkel und ohne deutliche Alula.

Der zweite Abschnitt der Costa ohne Borstchen unter der Behaarung.

Die zweite Längsader ungewöhnlich lang und an ihrem Ende zur dritten convergirend.

Die scchste Längsader fehlt. vortretendem Hinterwinkel und mit grofser Alula.

Der zweite Abschnitt der Cost a mit Borstchen unter der Behaarung.

Die zweite Längsader nur von gewöhnlicher Länge und an ihrem Ende zur dritten divergirend.

Die sechste Längsader ist vorhanden.

Es ist kaum zu begreifen, wie selbst noch in den neuesten Schriften, z. B. in Schiner's Fauna, so heterogene Arten in ein und dieselbe Gattung haben gebracht werden können.

Was nun die deutschen. zur Gattuug Balioptera gehörigen Arten betrifft, so giebt es deren 4, von welchen zwei sehr gemein, die andern beiden aber ziemlich gemein sind. Nach dem Erscheinen des sechsten Theils von Meigen's systematische. Beschreibung hat es wohl nur wenig deutsche Dipterologen gegeben, welche die 4 oben genannten Meigen'schen Namen nicht ohne alles Bedenken auf diese 4 bei uns häufigen Arten angewendet hätten. - Bal. combinata, venusta und tripunctata sind in den von Meigen gegebenen Beschreibungen, welche sich auf die gewöhnlichsten Varietäten derselben beziehen. gar nicht zu verkennen. Die vierte Art unterscheidet sich von Bal. combinata besonders durch die erheblich schmäleren Flügel und durch den gröfseren, die ganze Flügelspitze einnehmenden schwarzen Fleck. Ganz durch dieselben Merkmale unterscheidet Meigen aber seine $O p$. apicalis von combinata. Es würde nun gar kein Bedenken haben, diese vierte Art für die Meigen'sche apicalis zu erklären, wenn Meigen in der Beschreibung von apicalis nicht sagte: „Die beiden Queradern und die ganze Spitze braun". Es sind zwar bei der bei uns häufigen vierten Art beide Queradern wirklich braun, aber nur die hintere Querader ist schmal braun gesäumt. Vergleicht man Meigen's Beschreibung von apicalis mit seinen Beschreibungen der andern drei Arten, so ist die am nächsten liegende Interpretation des oben Angeführten die, dass er beide Queradern als braungesäumt habe bezeichnen wollen. Herr Zetterstedt hat sich dadurch bestimmen lafsen, eine auch bei uns zuweilen vorkommende Abänderung der Bal. combinata, bei welcher sich die dunkele Färbung der Flügelspitze bis über die Mündung der vierten Längsader hinaus erstreckt, für apicalis M eig. 
zu erklären, dabei aber offenbar nicht Gewicht genug darauf gelegl, dafs Meigen seiner" apicalis schmälere Flügel, als die der Bal. combinata sind, zuschreibt. Da nun dieses, keiner Mifsdeutung fähige plastische Merkmal auf keiue andere als die mehrfach erwähnte vierte der bei uns einheimischen Arten pafst, da bei dieser Art wirklich stets die ganze Flügelspitze dunkel gefärbt ist und da bei ihr die kleine Querader, besonders wenn man sie mit derjenigen der Bal. venusta vergleicht, wegen ihrer erheblich gröfseren Stärke und wegen ihrer gröfseren Dunkelheit viel mehr auffällt und bei weniger genauer Untersuchung wohl für schmal dunkelgesäumt gehalten werden kann, so habe ich kein Bedenken, der Meinung derjenigen beizutreten, welche unsere vierte, durch ihre schmalen Flügel ausgezeichnete Art für die ächte Meigen'sche apicalis halten.

Von den späteren Schriftstellern hat keiner zur Aufklärung über die Balioptera-Arten viel beigetragen. - Zuerst hat Herr Haliday als Opom. tremula eine Balioptera-Art beschrieben, in welcher die dunklere Abänderung der von Meigen nach helleren Exemplaren beschriebenen Bal. venusta gar nicht zu verkennen ist. - Herr Zetterstedt hat, wie ich schon bemerkte, irrthümlich eine seltenere Varietät der Bal. combinata für apicalis Meig. gehalten, die wahre Meigen'sche apicalis aber als Geom. terminalis beschrieben. - Auch Geom. angustipennis Zett. ist offenbar eine Balioptera; ob sie nur eine Varietät der Bal. venusta (worauf die Gestalt des dunkeln Flecks an der Flügelspitze und der Umstand, dafs die braune Säumung der hinteren Querader bei Bal. venusta oft sehr schmal und blafs ist, hinzudeuten scheinen), oder ob sie eine selbständige Art ist, müssen fernere Beobachtungen lehren. Dafs auch der Zetterstedt'schen Beschreibung von Geom. consobrina Exemplare von Balioptera-Arten zu Grunde gelegen haben, ist nicht zu bezweifeln; nach dem, was Herr Zetterstedt über die Gestalt des Flecks an der Flügelspitze sagt (,plerumque majuscula totum apicem occupans, raro parva et dimidiata" $\left.{ }^{6}\right)$, sollte man vermuthen, dafs er mehrere Arten mit einander vermengt habe. Das Uebrige sind Farbenangaben, aus denen bei der grofsen Veränderlichkeit der Färbung der Balioptera-Arten nicht viel zu machen ist; sie passen noch am besten auf helle Exemplare der Bal. tripunctata, bei der aber der Fleck an der Flügelspitze diese nie ganz einnimmt.

Aufser den 4 bei uns häufigen ist mir nur noch eine fünfte, südeuropäische Balioptera-Art vorgekommen, welche ich $\boldsymbol{B a l}$. ma juscula nennen will. Ich lasse die kurze Characteristik und Synonymie dieser 5 Arten hies folgen. 
1. Baliopt. combinata Linn. $\sigma$ \& 오. - Maculâ alarum apicali trigonâ limbisque venarum transversalium subangustis nigris, venae longitudinalis quartae apice nigro, cinereo-circumfuso. Long. corp. $1 \frac{5}{12}-1 \frac{7}{12}$ lin., long. al. $1 \frac{5}{1 \frac{2}{2}}-1 \frac{1}{2}$ lin.

Synon.? Musca combinata Linné, Syst. nat. XII. II. 997. 114.

Musca combinata Fabr., Syst. ent. 785. 65.; Spec. Ins. II. 451. 84.; Ent. Syst. IV. 352. 163.

Tephritis combinata Fabr., Syst. Antl. 317. 2.

Opomyza combinata Meig., Syst. Beschr. VI. 109. 18.

Geomyza combinata Zett., Ins. Lapp. 773. 1.; Dipt. Scand. VI. 2527. 1. var. a. u b.

Gelb, oben dunkler gelb; der Hinterleib des Männchens gewöhnlich mit alleiniger Ausnahme seiner Basis glänzend schwarz; der Hinterleib des Weibchens gewöhnlich mit vier ansehnlichen schwarzen Querbinden, welche am Hinterrande der einzelnen Abschnitte liegen; der verkürzte letzte Abschnitt desselben bald braungelb, bald schwarz. Beine ganz gelb, die Basis der Hinterschienen zuweilen etwas bräunlich. Die erste Längsader ist in der Gegend der Schulterquerader und oft auch gegen ihr Ende hin, wie bei den andern Arten, geschwärzt; die beiden Queradern haben mäfsig breite schwarze Säumung; an der Flügelspitze liegt ein dreieckiger braunschwarzer Fleck, weleher die dritte Längsader nur wenig überschreitet; das Ende der vierten Längsader ist schwarz gefärbt und von einer grauen oder mehr schwärzlichen Trübung umgeben; zwischen dieser Trübung und dem dreieckigen Flecke ist die Flügelfläche grauer gefärbt.

Der Hinterleib des Männchens zeigt zuweilen die sonst bei dem Weibchen gewöhnliche Färbung, während umgekehrt bei dunkelgefärbten Weibchen zuweilen der ganze Hinterleib mit Ausnahme der Basis glänzend schwarz gefärbt ist; bei hellgefärbten Weibchen fehlt die erste der schwarzen Binden zuweilen ganz, oder es sind auch wohl alle diese Binden nur kastanienbräunlich gefärbt. Die Breite der Säumung der Queradern ist ziemlich veränderlich, zuweilen so gering, dal's an der kleinen Querader kaum noch eine Spur derselben zu erkennen ist; umgekehrt ist die Verdunkelung um die Mündung der vierten Längsader zuweilen intensiver und durch eine grauschwärzliche Färbung mit dem dreieckigen Flecke verbunden.

A nmerkung. Linné's Diagnose seiner Musca combinata lautet: „Musca antennis setariis pilosa cinerea, abdomine nigro, alis margine tenuiore puncto nigro", und die Beschreibung: "Magniludo 
dupla Pediculi. Thorax cinereus. Abdomen nigrum. Alae complicatae fasciis duabus; posteriore magis atra, quae oritur a puncto majusculo nigro, ad marginem tenuiorem utriusque alae, versus posteriora." - Will man auch an dem "Thorax cinereus" keinen Anstofs nehmen, weil Linné mit , „cinereus" die verschiedensten Farben bezeichnel, so mufs man ihn doch billig an der auf unsere Arten gar nicht passenden Flügelbeschreibung nehmen und bekennen, dafs die bisher übliche Deutung des Linné'schen Namens durch seine Beschreibung nicht legitimirt ist. Da es bis jelzt nicht gelungen ist, eine andere in Schweden einheimische Fliegenart nachzuweisen, auf welche die Linné'sche Beschreibung von Musca combinata besser als auf gegenwärtige Art pafste, und da die älteren Autoren gar wohl uns unbekannte Auskünfte über Linné's Musca combinata gehabt haben können, so muls bis zu erbrachtem Gegenbeweise ihre Autorität für die Anwendung des Linné'schen Namens anf unsere Art maafsgebend bleiben.

2. Baliopt. venusta Meig. ठ̀ \& ․ - Maculâ alarum apicali minutâ trigonâ venaeque transversae posterioris limbo augusto nigris. - Long. corp. $1 \frac{1}{2}-1 \frac{7}{12}$ lin., long. al. $1 \frac{1}{2}$ lin.

Syn. Opomyza venusta Meig., Syst. Beschr. VI. 110. 20.

Geomyza combinata Zett., Dipt. Scand. V1. 2527. 1. var. c.

? Geomyza angustipennis Z ett., Dipt. Scand. VI. 2529. 2. Opomyza tremula Hal., Ent. Mag. I. 177.

Kopf gelb, nur bei sebr blassen Exemplaren die Fühler fast weifsgelblich; bei dunklen Exemplaren ist der obere Theil des Scheiteldreiecks und der breiten Seitenleisten der Stirn oft gebräunt. Thorax bei blassen Exemplaren rothgelb, bei dunklen gelbbraun oder braunscbwärzlich, doch bleiben auch bei diesen der Vorderrand und die Hinterecken der Oberseite meist, und der vordere und untere Theil der Brustseiten stets hellgefärbt. Das Schildchen ist bei hellen Stücken gelb oder rothgelb, bei dunklen nur am Rande roth, sonst aber braunschwärzlich gefärbt. Hinterleib glänzend schwarz, höchstens an der alleräufsersten Basis schmal gelbroth; die Spitze des letzten Hinterleibsabschnitts ist bei dem Weibchen in veränderlicher Ausdehnung röthlich oder weifslich gefärbt. Die Beine sind bei sehr hellen Exemplaren ganz gelb, nur die Hinterschienen gegen die Wurzel hin ganz schwach bräunlich; bei dunklen Exemplaren sind dagegen die Hinterschienen gewöhnlich bis fast zur Spitze dunkelbraun gefärbt und die Hinterschenkel auf ibrer Mitte in grofser Ausdehnung stark gebräunt. Sehr kenntlich ist die Art durch ihre 
Flügelzeichnung; bei ausgefärbten Exemplaren ist die Costalzelle stets viel deutlicher schwarz ausgefüllt, als bei allen andern mir bekannten Arten; die kleine Querader ist weder verdickt, noch geschwärzt, noch dunkel gesäumt; der an der Flügelspitze liegende dreieckige braunschwarze Fleck ist klein und überschreitet die dritte Längsader etwas; das Ende der vierten Längsader ist nie von dunkler Trübung umgeben und die hintere Querader stets nur schmal braunschwarz gesäumt. - Bei ganz hellen Exemplaren ist zuweilen die schwarze Ausfüllung der Costalzelle minder vollständig.

Anmerkung. In Haliday's Beschreibung von Opom. tremula ist eine dunklere Abänderung der Bal. venusta meines Erachtens gar nicht zu verkennen. Wenn er die Flügel spitzer als bei $\boldsymbol{B a l}$. tripunctala nennt, so meint er damit offenbar nichts anderes, als dafs sie nach der Basis hin etwas mehr zugespitzt seien, was auch wirklich der Fall ist, und wenn er die Flügelzeichnung mit derjenigen von Mycetaulus bipunctatus vergleicht, so hat er dabei offenbar die schwarze Ausfüllung der Costalzelle und die Gestalt des Spitzenflecks im Auge gehabt, welche wirklich diese Vergleichung vollständig rechtfertigen; dafs die bei Mycetaulus bipunctatus nicht dunkel gefärbte hintere Querader bei seiner Opom. tremula dunkel gesäumt ist, sagt er ausdrücklich. - Zetterstedt's Geom. combinata var. c. gehört offenbar hierher. Ob seine Geom. angustipennis sich, wie ich vermuthe, als eine Abänderung gegenwärtiger Art oder ob sie sich als selbstständige Art ausweisen werde, bedarf noch der Entscheidung; Grund zu meiner Vermuthung geben mir Exemplare der Bal. venusla, bei denen die Säumung der hinteren Querader so geringfügig ist, dafs man diese Ader, anstatt sie als ,äufserst schmal schwarz gesäumt“" zu bezeichnen, fast ebenso gut „blos schwarz gefärbt" nennen könnte. Uebrigens spricht sich Herr Zetterstedt selbst über die Artrechte seiner Geom. angustipennis etwas zweifelhaft aus.

3. Baliopt. apicalis Meig. $\delta \&$ ㅇ. - Alarum angustissimarum apice toto venaeque transversalis posticae limbo angusto nigris. - Long. corp. $1_{\frac{1}{12}}$ lin., long. al. $1 \frac{1}{3}$ lin.

Syn. Opomyza apicalis Meig., Syst. Beschr. VI. 109. 19.

Tephritis maculata A hr., Faun. III. 22.

Geomyza terminalis Zett., Dipt. Scand. VI. 2533. 8.

Kopf und Thorax gelb oder gelbroth; bei etwas dunkleren Exemplaren pflegt wenigstens der obere Theil des Hinterkopfes und der Hinterrücken gebräunt zu sein, während bei sehr dunkeln der 
oberste Theil der Stirn und des Hinterkopfs, der obere Theil der Brustseiten, der gröfste Theil der Oberseite des Thorax und das Schildchen schwarzbraun sind, der Hinterrücken aber eine ganz schwarze Färbung hat. Hinterleib glänzend schwarz, der kurze erste Abschnitt bei hellen Exenplaren rothgelb, bei dunkleren braun, zuweilen ganz schwarz; das Ende des lelzten Abschnitts des weiblichen Hinterleibs ist, wie bei den anderen Arten, in verschiedener Ausdehnung röthlich oder weifslich gefärbt. Die Beine sind bei hellen Exemplaren ganz gelb; bei dunkelen zeigt sich an der Wurzel der Hinterschienen jenseit der Mitte der Hinterschenkel eine deutliche, zuweilen ziemlich dunkele Bräunung; zuweilen sind dieselben Stellen auch an den Mittelbeinen gebräunt, doch ist die Bräunung derselben dann stets viel weniger intensiv. Die Flügel sind etwas kürzer und viel schmäler, als bei den anderen Arten; hinsichtlich der schwarzen Ausfüllung der Costalzelle kömmt diese Art der Bal. venusta am nächsten; die kleine Querader ist ziemlich stark und hat eine dunkelbraune Färbung, aber keine dunkelbraune Säumung; der Fleck an der Flügelspitze ist verhältnifsmäfsig grofs und nimmt diese stels ganz ein, so dafs die Mündungen der zweiten, dritten und vierten Längsader innerhalb desselben liegen; die kurze und dem Hinterrande des Flügels noch mehr genäherte hintere Querader hat eine schmale schwarzbraune Säumung.

Anmerkung. Das Citat aus der Fauna von Ahrens vermag ich augenblicklich nicht zu vergleichen, kann also auch seine Richtigkeit nicht verbürgen. - Zetterstedt hat Meigen's Opom. apicalis für eine zuweilen vorkommende Abänderung der Bal. combinata gehalten, bei welcher die graue Trübung zwischen dem Spitzenflecke und der die Mündung der vierten Längsader umgebenden Trübung intensiver als gewöhnlich auftritt und beide mit einander verbindet; diese Deutung der Meigen'schen Beschreibung ist aber unzulässig, weil Meigen ausdrücklich angiebt, dafs die Flügel seiner Opom. apicalis schmäler als die seiner Opom. combinata seien. - Zetterstedt's Angaben über seine Geom. terminalis leiden an einem Versehen; er sagt von ihrem Flügelgeäder: ,nervi transversi modice remoti, horum ordinarius ad marginem alae interiorem retractus, leniter infuscatus, medius paullo pone nervum auxiliarem omnium brevissimum ductus." Da die hierin enthaltenen Angaben einander widersprechen, mufs nothwendig eive derselben falsch sein. Der Umstand, dafs die Zetterstedt'sche Beschreibung in allem Uebrigen vollständig auf gegenwärtige, aber auf keine andere mir bekannte Art pafst, berechtigt mich zu der Vermuthung, dafs seine Angabe über die Stellung 
der kleinen Querader zur Mündung der ersten Längsader (nervus uuxiliaris $\mathrm{Z}$ ett.) diese falsche Angabe sei; ist sie es, so unterliegt die Identität von Geom. terminalis und Bal. apicalis wohl keinem Zweifel. -

4. Baliopt. tripunctata Fall. $\delta \&$ f. - Maculâ alarum apicali trigonâ, venarum transversalium limbis latissimis et puncto in imâ cellulae submarginalis basi nigris.

Syu. Geomyza tripunctata Fall., Geom. 2. 2.

Opomyza tripunctata MI eig., Syst. Beschr. VI. 110. 21.

Geomyza tripunctata Zett., Ins. Lapp. 774. 2.; Dipt. Scand. 2531. 5.

Sie ist eine in ihrer Körperfärbung ganz besonders veränderliche Art. Bei den hellsten Exemplaren sind Kopf und Thorax mit alleiniger Ausnahme des auch bei ihnen gewöhnlich braun gefärbten Hinterrückens, so wie der erste und die Vorderhälfte des zweiten Hinterleibsabschnittes gelb oder rothgelb, der übrige Hinterleib schwarz und die ganzen Beine bellgelb gefärbt. Bei den dunkelsten Exemplaren sind dagegen der obere Theil des Scheiteldreiecks und der Seitenleisten der Stirn, der Hinterkopf mit Ausnahme seines Unterrandes, der ganze Thorax und der ganze Hinterleib schwarz; selbst der weifsliche Fleck, welcher sich auch bei dieser Art am Ende des letzten Abschnitts des weiblichen Hinterleibs befindet, ist völlig verdunkelt und nur an seiner hellen Pubescenz kenntlich; die ganze Hinterseite der Vorderschenkel, die hinteren Schenkel mit Ausnahme der Spitze und Wurzel und die Hinterschienen sind bei ihnen braun. Diese beiden Extreme sind durch die allmäligsten Uebergänge mit einander verbunden. Bei uns sind Exemplare der dunkelsten Abänderung sehr selten, bei weitem am häufigsten solche, welche zwischen beiden Extremen die Mitle halten. während die sicilischen, griechischen und kleinasiatischen Exemplare meiner Sammlung der Mehrzahl nach zu den dunkelsten Abänderungen gehören. - Trotz der grofsen Veränderlichkeit in der Körperfärbung ist diese Art doch, wie die anderen, an ihrer eigenthümlichen Flügelzeichnung leicht zu erkennen. Beide Queradern haben nämlich eine äufserst breite und weit über die durch sie verbundenen Längsadern hinausgreifende braunschwarze Säumung, namentlich reicht die Säumung der klcinen Querader fast vollständig von der zweiten bis zur fünften Längsader; der dreieckige, braunschwarze, die dritte Längsader etwas überschreitende Spitzenfleck hat etwa die Grölse und Gestalt wie bei Bal. combinata, die Mündung der vierten 
Längsader ist aber von keiner dunkelen Trübung umgeben; sehr charakteristisch ist eine braunschwärzliche, doch minder dunkele Trübung, welche sich von der Spitze der Costalzelle bis auf die äufserste Basis der Submarginalzelle hinzieht, da sie bei keiner anderen bisher bekannt gewordenen Art vorhanden ist; innerhalb derselben sind die zweite und dritte Längsader dunkel gefärbt.

5. Baliopt. majuscula nov. sp. ㅇ. - Reliquis speciebus major, maculà alarum apicali trigonà venarumque transversalium

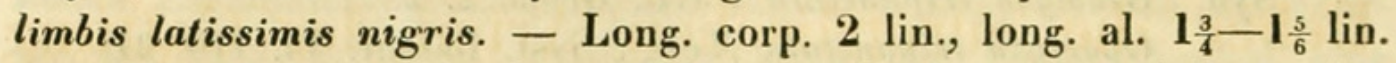

Diese schöne, durch erheblichere Gröfse ausgezeichnete Art gleicht am meisten der Baliopt. tripunctata. Ob sie in der Körperfärbung ebenso veränderlich wie diese ist, vermag ich nicht zu sagen. Bei meinen beiden Weibchen sind Kopf und Thorax gelb. roth, nur der Hinterrücken dunkelbraun. Hinterleib etwas breiter als bei den anderen Arten, glänzend schwarz, nur an der alleräufsersten Basis mit der Spur von rother Färbung; der weifsliche Fleck an der Spitze des letzten Abschnitts etwas undeutlich. Beine gelblich, nur die Basis der Hinterschienen, obwohl in geringer Ausdehnung, doch sehr intensiv braun. Spitzenfleck der Flügel und die dunkele Säumung der Queradern wie bei Baliopt. tripunctata, nur die Säumung der kleinen Querader nicht ganz so breit und viel weniger über die durch sie verbundenen Queradern hinausgreifend; von der für Baliopt. tripunctata so charakteristischen, sich von der Spitze der Costalzelle auf die äufserste Basis der Submarginalzelle hinziehenden Trübung ist keine Spur zu bemerken.

$\mathrm{Z}$ usatz. Von den bereits beschriebenen exotischen Arten gehört Opom. lurida Loew aus Sitka zur Gattung Balioptera. Sie ähnelt in der Flügelzeichnung am meisten der Baliopt. combinata, doch ist der Spitzenfleck etwas kleiner, namentlich auf seinem vorderen Theile weniger ausgebreitet, und der hinter der fünften Längsader liegende Abschnitt der Flügelfläche ist breiter. Die Färbung von Kopf und Thorax scheint in derselben Weise veränderlich zu sein, wie bei Baliopt. tripunctata. Der Hinterleib ist bei beiden Geschlechtern glänzend schwarz mit kaum bemerkbarer Spur von rother Färbung an seiner alleräufsersten Basis und bei dem Weibchen mit dem gewöhnlichen schmutzig weifslichen oder rothgelblichen Flecke auf dem Ende des letzten Abschnitts. Vor dem Ende der Hinterschenkel und an der Wurzel der Hinterschienen zeigt sich mehr oder weniger deutliche Bräunung. Baliopt. lurida ist $1 \frac{2}{3}-1 \frac{3}{4}$ Linien lang und die Flügel messen $1 \frac{2}{3}$ Linien, so dafs sie an Gröfse nur von Baliopt. majuscula übertroffen wird. 


\section{$2 \mathrm{BHL}$ Biodiversity Heritage Library}

1864. "Die Arten der Gattung Balioptera." Berliner entomologische Zeitschrift I herausgegeben von dem Entomologischen Vereine in Berlin 8(3प4)347-356. https://doi.org/10.1002/mmnd.18640080313.

View This Item Online: https://www.biodiversitylibrary.org/item/36390

DOI: https://doi.org/10.1002/mmnd.18640080313

Permalink: https://www.biodiversitylibrary.org/partpdf/210026

\section{Holding Institution}

Smithsonian Libraries

\section{Sponsored by}

Smithsonian

\section{Copyright \& Reuse}

Copyright Status: Public domain. The BHL considers that this work is no longer under copyright protection.

This document was created from content at the Biodiversity Heritage Library, the world's largest open access digital library for biodiversity literature and archives. Visit BHL at https://www.biodiversitylibrary.org. 